\title{
The association between low birth weight and dental caries among 11-to-13-year-old school age children in Ningbo, China
}

\author{
Xiaoyan Weng ${ }^{1,2 \dagger}$, Yiting Lou ${ }^{1,2+}$, Ran Tao ${ }^{3}$, Yongzheng Li ${ }^{2}$, Danna CaO ${ }^{1,2^{*}}$, Mengfei Yu ${ }^{2 *}$, Binbin Ying ${ }^{1,2^{*}}$ and \\ Huiming Wang ${ }^{2}$
}

\begin{abstract}
\section{Introduction}

Low birth weight (LBW) risk factors, such as maternal diabetes, hypertension, obesity, and older age, have been on the rise, making LBW a major public health issue in China [1]. A 2013 epidemiological survey showed that the incidence of LBW (common in preterm babies) in China was $6.1 \%(<2500 \mathrm{~g})$ [2]. Advances in technology have decreased the mortality rate due to LBW. However, complications, such as respiratory disorders and oral problems, have been reported in patients with LBW [3,
\end{abstract}

Background: The association between low birth weight (LBW) and dental caries is currently unclear. The aim of this study was to investigate the association of LBW with dental caries in permanent teeth in children of Ningbo city.

Methods: A total of 1975 children aged 11-to-13years in Ningbo, China were enrolled in this cross-sectional study. LBW was defined as a birthweight $<2500 \mathrm{~g}$. Ten dentists assessed the status of dental caries in permanent teeth in line with the World Health Organization (WHO) criteria and guidelines. Decayed, missing or filled teeth were considered to have dental caries. Parental questionnaires were used to collect child information. Non-conditional logistic regression analysis was used to estimate odds ratios (ORs) and the corresponding 95\% confidence intervals (Cls).

Results: Dental caries in permanent teeth was found in 610 children (30.9\%), with a mean DMFS of 2.09 (SD=1.2). The adjusted ORs for dental caries in permanent teeth was $1.46(95 \% \mathrm{Cl} 1.00,2.13)$ for LBW.

Conclusions: LBW was not associated with dental caries in permanent teeth in the study population.

Keywords: Low birth weight, Dental caries, Permanent teeth, Children, China

\footnotetext{
*Correspondence: caocao1985114@126.com; yumengfei@zju.edu.cn; yingbinbin@zju.edu.cn

${ }^{\dagger}$ Xiaoyan Weng and Yiting Lou contributed equally to this work.

${ }^{2}$ The Affiliated Hospital of Stomatology, School of Stomatology, Zhejiang University School of Medicine, and Key Laboratory of Oral Biomedical Research of Zhejiang Province, Zhejiang 310006, Hangzhou, China Full list of author information is available at the end of the article
}

4]. Previous studies found that LBW and preterm children result in poor dental health and hygiene [4].

The incidence of dental caries is influenced by genetic, socioeconomic and early life factors [5-8]. Prematurity and LBW have been found to only affect the enamel structure of the primary teeth since permanent teeth mineralize only after birth. In recent years, some studies have shown that birth outcomes affect the ameloblasts during the secretory or maturation phase of tooth development, causing both hypoplastic and hypomineralized enamel [9-12]. The defects affect the permanent incisors and first molars during permanent dentition. To date, the association of LBW with dental caries is unclear $[4,13-$ 16]. Furthermore, as far as we know, no study has investigated this in China.

Therefore, we investigated the association of LBW with dental caries in permanent teeth of Chinese children. 


\section{Methods}

\section{Design}

The following formula was used to calculate the required sample size:

$$
n=\operatorname{deff} \frac{\mu^{2} p(1-p)}{\varepsilon^{2}}
$$

Where $n$ is the sample size, deff is the design effect set as $1.5, p$ is the prevalence of dental caries, $\mu$ is the level of confidence, and $\varepsilon$ is the margin of error. The prevalence of dental caries is $28.9 \%$ according to the Third National Oral Survey of China. The non-response rate is sets as $20 \%$. Based on this estimation, the final sample size was 1813 .

The study was conducted on 11-to-13-year-old children from 10 regions involved in the pit and fissure closure treatment in 2020, public welfare activity for school-age children in Ningbo city. Pit and fissure closure treatment prevents dental caries, mainly in the pits and fissures of occlusal tooth surfaces. The participants were recruited from each region, using a stratified sampling method. LBW was defined as having a birth weight $<2500 \mathrm{~g}$, as proposed by Mikolajczyk [17]. The children were diagnosed with caries in permanent teeth if the permanent incisors or first molars were decayed, missing, or filled [18]. Written informed consent was obtained from all children and parents. The study was conducted in line with Studies in Epidemiology (STROBE) guidelines for reporting observational studies [19].

\section{Oral examination}

Ten dentists from The Affiliated Ningbo Hospital of Zhejiang University performed the clinical examinations on the children. A visual examination using optimal lighting, a mouth mirror, and a probe in a supine position (no X-rays) was carried out to assess dental caries. Teeth were dried before the inspection to guarantee accurate results. Dental caries was measured using decayed, missing and filled surfaces (DMFS) following the WHO criteria [20].

\section{Quality control}

An experienced dentist (B.Y) trained the ten examiners to equip them with theoretical and clinical knowledge before examination. Each examiner was calibrated with the standard examiner (B.Y) and other examiners by assessing 50 children on each of 2 occasions, 1 month apart. These 50 children were enrolled in this study and were randomly selected. Additionally, $5 \%$ of the samples were randomly reexamined to monitor interexaminer and intra-examiner reproducibility.

\section{Questionnaire survey}

A structured questionnaire was used to collect information such as age, gender, birth weight, dental health behavior (the frequency of sugary food intake, toothbrushing frequency, age at first brushing, regular dental check-ups, fluoride use), the maternal exposure to tobacco smoke during pregnancy, parental educational level.

Fluoride use was classified as positive when children used fluoride agents at home, such as toothpaste and gel, or topical fluoride products at hospital. Birth weight was filled according to the Maternal and Child Health Handbook (booklet for pregnancy, delivery and postnatal/child health) issued by the Zhejiang Province Department of Health.

\section{Statistical analysis}

Chi-square or Fisher's exact tests were used to compare subjects' characteristics. Gender, parental educational level, maternal exposure to tobacco smoke during pregnancy, the frequency of sugary food intake, toothbrushing frequency, age at first brushing, fluoride use, regular dental check-ups were considered confounding factors based on existing literature. Non-conditional logistic regression analysis was used to estimate crude and adjusted odds ratios (ORs) with 95\% confidence intervals (95\%CIs) to determine the association of LBW with dental caries. DMFS was the dependent variable for the non-conditional logistic regression analysis. "DMFS $=0$ " was denoted as " 0 ", and "DMFS $>0$ " was denoted as " 1 ". The SAS software Version 9.4 (SAS Institute, Cary, North Carolina, USA) was used for all statistical analysis.

\section{Results}

A total of 8702 11-to-13-year-old school age children received pit and fissure closure and their parents were provided with a questionnaire. Two thousand one questionnaires subsequently recruited from 10 regions, using a stratified sampling method (23.0\%). Missing answers and illogical data were resolved by contacting parents via a telephone interview. A total of 1975 children (22.7\%) with complete information were enrolled. The Kappa values used to assess inter-examiner and intra-examiner reproducibility ranged from 0.81 to 0.96 .

The mean age of the 1975 children was $11.3(\mathrm{SD}=0.5)$. Among them were 1013 (51.2\%) males and 962 (48.8\%) females. A total of 610 children (30.9\%) had dental caries in permanent teeth. Specifically, the prevalence of dental caries in LBW children and normal birth weight 
(NBW) children was 38.3 and $30.3 \%$, respectively. The mean DMFS of all subjects was $0.65(\mathrm{SD}=1.2)$, and the mean DMFS of LBW children and NBW children was $0.84(\mathrm{SD}=1.3)$ and $0.63(\mathrm{SD}=1.2)$, respectively.

Table 1 Distribution of the sample and the population in the 10 regions of Ningbo

\begin{tabular}{llllll}
\hline & \multicolumn{2}{l}{ Numbers of sample } & \multicolumn{2}{l}{$\begin{array}{l}\text { Number of } \\
\text { Population by } \\
\text { Region }\end{array}$} \\
\cline { 2 - 3 } \cline { 5 - 6 } & (n) & $\%$ & & & \\
\cline { 5 - 6 } Beilun & 609 & 7.0 & & 429,700 & 7.1 \\
Cixi & 1638 & 18,8 & & 1059,600 & 17.4 \\
Fenghua & 673 & 7.7 & & 480,600 & 7.9 \\
Haishu & 951 & 10.9 & & 633,500 & 10.4 \\
Jiangbei & 378 & 4.3 & & 262,800 & 4.3 \\
Ninghai & 877 & 10.1 & & 633,900 & 10.4 \\
Xiangshan & 747 & 8.6 & & 560,400 & 9.2 \\
Yingzhou & 1376 & 15.8 & & 931,000 & 15.3 \\
Yuyao & 1071 & 12.3 & & 835,900 & 13.7 \\
Zhenhai & 382 & 4.4 & & 257,600 & 4.3 \\
\hline
\end{tabular}

The sample and the population distributions in the 10 Ningbo Province regions are shown in Table 1. The characteristics of the study population are prescribed in Table 2 . The mean birth weight of the entire study sample was 3, $332.1 \mathrm{~g}$, and the prevalence of LBW was $6.5 \%$. About $70 \%$ of the subjects consumed sugary foods less than once a day, and more than $40 \%$ were over 3 years old before starting to brush. About $74 \%$ of children brushed teeth $\geq 2$ times daily, and $70 \%$ received dental examination at least once a year. Approximately $54 \%$ of the children used fluoride agents. Of note, most dietary habits and lifestyles were similar between LBW children and NBW children.

The association of LBW with dental caries in permanent teeth is shown in Table 3. There was no association between LBW and dental caries in permanent teeth after adjusting for confounding factors.

\section{Discussion}

In this cross-sectional study, LBW was not associated with dental caries. This is consistent with findings in a retrospective cohort research in the USA, which found no increased risk of caries in very low birth weight (VLBW) group [3]. However, the study in USA did not distinguish posteruptive

Table 2 Distribution of selected characteristic in 1, 975 children

\begin{tabular}{|c|c|c|c|c|c|c|c|c|}
\hline & \multirow[b]{2}{*}{ Category } & \multicolumn{2}{|c|}{$\begin{array}{l}\text { Low birth } \\
\text { weight }\end{array}$} & \multicolumn{2}{|c|}{$\begin{array}{l}\text { Normal birth } \\
\text { weight }\end{array}$} & \multicolumn{2}{|c|}{ Total } & \multirow[b]{2}{*}{$P$} \\
\hline & & (n) & $\%$ & (n) & $\%$ & $(n)$ & $\%$ & \\
\hline \multicolumn{9}{|l|}{ Variable } \\
\hline Total & & 128 & 6.5 & 1847 & 93.5 & 1975 & 100 & \\
\hline \multirow[t]{2}{*}{ Sex } & Female & 61 & 47.7 & 901 & 48.8 & 962 & 48.1 & .805 \\
\hline & Male & 67 & 52.3 & 946 & 51.2 & 1013 & 51.3 & \\
\hline \multirow[t]{3}{*}{ Paternal educational level (years) } & $<9$ & 47 & 36.7 & 745 & 40.3 & 792 & 40.1 & .407 \\
\hline & $9-12$ & 45 & 35.2 & 546 & 29.6 & 591 & 29.9 & \\
\hline & $>12$ & 36 & 28.1 & 556 & 30.1 & 592 & 30.0 & \\
\hline \multirow[t]{3}{*}{ maternal exposure to tobacco smoke during pregnancy } & Active smoking & 0 & 0.0 & 16 & 0.8 & 16 & 0.8 & $<.001$ \\
\hline & Passive smoking & 62 & 48.4 & 141 & 7.6 & 203 & 10.3 & \\
\hline & No smoking & 66 & 51.6 & 1690 & 91.5 & 1756 & 88.9 & \\
\hline \multirow[t]{3}{*}{ The frequency of sugary food intake (times/day) } & $<1$ & 90 & 70.3 & 1286 & 69.6 & 1376 & 69.7 & .782 \\
\hline & $1-2$ & 28 & 21.9 & 383 & 20.7 & 411 & 20.8 & \\
\hline & $>2$ & 10 & 7.8 & 178 & 9.6 & 188 & 9.5 & \\
\hline \multirow[t]{3}{*}{ Age at first brushing (years old) } & 2 & 29 & 22.7 & 480 & 26.0 & 509 & 25.8 & .065 \\
\hline & 3 & 35 & 27.3 & 635 & 34.4 & 670 & 33.9 & \\
\hline & $>3$ & 64 & 50.0 & 732 & 39.6 & 796 & 40.3 & \\
\hline \multirow[t]{3}{*}{ Toothbrushing frequency (times/day) } & 0 & 1 & 1.0 & 18 & 1.0 & 19 & 1.0 & .398 \\
\hline & 1 & 38 & 21.9 & 450 & 24.4 & 488 & 24.7 & \\
\hline & $>2$ & 89 & 60.5 & 1379 & 74.7 & 1468 & 74.3 & \\
\hline \multirow[t]{3}{*}{ Dental check-ups (times/year) } & 0 & 38 & 30.0 & 476 & 25.8 & 514 & 26.0 & .583 \\
\hline & 1 & 57 & 44.5 & 842 & 45.6 & 899 & 45.5 & \\
\hline & 2 & 33 & 25.8 & 529 & 28.6 & 562 & 28.5 & \\
\hline Fluoride use & & 53 & 41.4 & 1016 & 55.0 & 1069 & 54.1 & .003 \\
\hline
\end{tabular}


Table 3 ORs and 95\% Cls for the association between dental caries in permanent teeth and LBW

\begin{tabular}{lllll}
\hline & & Prevalence & Unadjusted ORs [95\%Cl] & Adjusted ORs [95\%Cl] \\
\hline \multirow{2}{*}{ Low birth weight } & Yes & $49 / 128(38.3 \%)$ & $1.43[0.96,2.06]$ & $1.46[1.00,2.13]$ \\
& No & $560 / 1847(30.3 \%)$ & 1.00 & 1.00
\end{tabular}

ORs odds ratios, 95\% Cl 95\% confidence interval

Adjusted for gender, paternal educational level, maternal exposure to tobacco smoke during pregnancy, the frequency of sugary food intake, age at starting to brush, toothbrushing frequency, dental check-up, use of fluoride

enamel loss in hypomineralized areas from caries in permanent molars and incisors. A previous retrospective cohort study conducted in Dubai found different results. It was found that enamel defects and dental caries in permanent teeth were significantly higher in the preterm group than in the full-term group in 5-to-10-year-old children [21]. Hypomineralization has been found to increases susceptibility to dental caries. The relationship between LBW and hypomineralization is currently unclear. A cross-sectional study involving 4208 years old children in Thailand reported that LBW was not associated with molar incisor hypomineralization [22] while other similar studies found an association between the two [23, 24].

Several factors may explain these inconsistencies. For instance, some studies may have examined for dental caries at an age when dental caries had not developed, hence the association of LBW and dental caries was not detected $[25,26]$. Therefore, the age of caries assessment should be investigated in future studies. Kay [27] reported that dental caries should be assessed at least 2 years after teeth eruption, to provide enough time for teeth decay. The inconsistencies may also be caused by different methodologies across studies. Nondifferential measurement error cannot be avoidable in a cross-sectional study. Furthermore, different confounding factors, which limits the feasibility of inter-study comparisons. Therefore, findings from previous studies are inconsistent and cannot be used to draw a clear conclusion applicable to all populations.

This study examined the relationship between LBW and dental caries in permanent teeth among children aged 11-13 years. Previous investigations focused on the influence of LBW on primary teeth $[13,21,28]$. The impacts of LBW on permanent teeth, especially permanent incisors and first molars, primarily originate from the secretory phase of the ameloblast. It begins in utero, and the maturation phase starts at birth. If inadequate mineralization or trauma occurs during these periods, the enamel defects appear, increasing the risk of caries [3, 29].

Several factors may explain the lack of association between LBW and dental caries in permanent teeth. LBW babies receive more attention and adequate nutritional intake from guardians than NBW infants [30]. Besides, their guardians are more proactive in implementing health and oral health instructions, consistent with findings by Gravina [31]. Specifically, Gravina [31] showed that premature children have lower rates of caries than term children because of appropriate oral health care and regular pediatric care. Furthermore, premature birth and LBW cause delayed teeth eruption, reducing caries information, thus minimizing the risk of dental caries [30,32].

This study has several advantages. The participants were homogeneous in terms of age and geographic background. Detailed information, such as the frequency of sugary food intake, the frequency of brushing and starting age, regular dental check-ups were collected, making it easier to control for confounding factors. Birth weight was obtained from the hospital health booklet, suggesting that the data was accurate. Besides, the study included a large number of participants, which reduces contingency.

Nevertheless, there are some limitations worth mentioning. The outcomes may vary across different populations due to various cultural and social factors. The participants were from an economically developed area, and hence the present findings may not be applicable to other sites. Moreover, some limitations of a cross-sectional study such as selection bias and recall bias, could not be avoided. Furthermore, we did not assess the small for gestational age (SGA) that precisely evaluates intrauterine growth restriction [33].

\section{Conclusion}

In this cross-sectional study, LBW was not associated with dental caries in permanent teeth in children from Ningbo, Zhejiang province. However, it is likely that such an association exists in other areas. Further prospective studies should be conducted to verify the relationship between the LBW and dental caries in permanent teeth.

\section{Abbreviations}

LBW: Low Birth Weight; WHO: World Health Organization; ORs: Odds ratios; 95\% Cls: 95\% Confidence Intervals; NBW: Normal Birth Weight; VLBW: Very Low Birth Weight; SGA: Small for Gestational Age.

\section{Acknowledgements}

All authors would like to thank the children and their parents for their active participation in this study.

\section{Authors' contributions}

$X . W$ and $Y . L$ wrote the main manuscript text, R. T and $Y . L$ did the statistical analysis, X. W, Y. L, D. C, B. Y, M. Y, H. W conceived and designed the study. 
All authors read and approved the final manuscript and contributed to the preparation of this manuscript.

\section{Funding}

This study was financed in part by the National Natural Science Foundation of China, Grant number: 81873720, and the Medical Science and Technology Planning Project of Ningbo City, Zhejiang Province, Grant number: 2020 Y05.

\section{Availability of data and materials}

The data from this study is available with the corresponding authors and can be accessed on request.

\section{Declaration}

\section{Ethics approval}

All methods in this study were performed in accordance with the principles of the relevant guidelines and regulations of Declaration of Helsinki, and The Affiliated Ningbo Hospital of Zhejiang University Medical Ethics Committee (2020-R280) approved the study.

All authors are in agreement with the content of the manuscript. Written informed consent was obtained from all children and parents.

\section{Consent for publication}

Not applicable.

\section{Competing interests}

We declare to have no competing interests.

\section{Author details}

${ }^{1}$ The Department of Stomatology, The Affiliated Ningbo Hospital of Zhejiang University, Ningbo 315000, China. ${ }^{2}$ The Affiliated Hospital of Stomatology, School of Stomatology, Zhejiang University School of Medicine, and Key Laboratory of Oral Biomedical Research of Zhejiang Province, Zhejiang 310006, Hangzhou, China. ${ }^{3}$ The Department of Human Anatomy, Laboratory of Clinical Applied Anatomy, School of Basic Medical Science, Fujian Medical University, Fuzhou 350000, China.

Received: 30 March 2021 Accepted: 20 October 2021 Published online: 04 November 2021

\section{References}

1. Rao J, Fan D, Wu S, et al. Trend and risk factors of low birth weight and macrosomia in South China, 2005-2017: a retrospective observational study. Sci Rep. 2018;8(1):3393.

2. Chen Y, Li G, Ruan Y, Zou L, Wang X, Zhang W. An epidemiological survey on low birth weight infants in China and analysis of outcomes of fullterm low birth weight infants. BMC Pregnancy Childbirth. 2013;13:242.

3. Nelson S, Albert JM, Lombardi G, et al. Dental caries and enamel defects in very low birth weight adolescents. Caries Res. 2010;44(6):509-18.

4. Darlow BA, Horwood LJ, Woodward LJ, et al. The New Zealand 1986 very low birth weight cohort as young adults: mapping the road ahead. BMC Pediatr. 2015;15:90

5. Andre Kramer AC, Petzold M, Hakeberg M, Ostberg AL. Multiple socioeconomic factors and dental caries in Swedish children and adolescents. Caries Res. 2018:52(1-2):42-50.

6. Sun X, Bernabe E, Liu X, Gallagher JE, Zheng S. Early life factors and denta caries in 5-year-old children in China. J Dent. 2017:64:73-9.

7. Silva MJ, Kilpatrick NM, Craig JM, et al. Genetic and Early-Life Environmental Influences on Dental Caries Risk: A Twin Study. Pediatrics. 2019:143:5.

8. Shungin D, Haworth S, Divaris K, et al. Genome-wide analysis of dental caries and periodontitis combining clinical and self-reported data. Nat Commun. 2019;10(1):2773.

9. Suckling GW. Developmental defects of enamel--historical and presentday perspectives of their pathogenesis. Adv Dent Res. 1989;3(2):87-94

10. Sabel N, Johansson C, Kühnisch J, et al. Neonatal lines in the enamel of primary teeth--a morphological and scanning electron microscopic investigation. Arch Oral Biol. 2008;53(10):954-63.
11. Alaluusua S. Aetiology of molar-incisor Hypomineralisation: a systematic review. Eur Arch Paediatr Dent. 2010;11(2):53-8.

12. Sönmez H, Yıldırım G, Bezgin T. Putative factors associated with molar incisor hypomineralisation: an epidemiological study. Eur Arch Paediatr Dent. 2013;14(6):375-80

13. Tanaka K, Miyake Y. Low birth weight, preterm birth or small-for-gestational-age are not associated with dental caries in young Japanese children. Bmc Oral Health. 2014;14:38.

14. Brogårdh-Roth S, Matsson L, Klingberg G. Molar-incisor hypomineralization and oral hygiene in 10- to-12-yr-old Swedish children born preterm. Eur J Oral Sci. 2011;119(1):33-9.

15. Cruvinel VR, Gravina DB, Azevedo TD, Rezende CS, Bezerra AC, Toledo OA. Prevalence of enamel defects and associated risk factors in both dentitions in preterm and full term born children. J Appl Oral Sci. 2012;20(3):310-7.

16. Bernabé E, MacRitchie H, Longbottom C, Pitts NB, Sabbah W. Birth weight, breastfeeding, maternal smoking and caries trajectories. J Dent Res. 2017;96(2):171-8.

17. Mikolajczyk RT, Zhang J, Betran AP, et al. A global reference for fetalweight and birthweight percentiles. Lancet. 2011;377(9780):1855-61.

18. Castro ALS, Vianna MIP, Mendes CMC. Comparison of caries lesion detection methods in epidemiological surveys: CAST. ICDAS and DMF Bmc Oral Health. 2018:18.

19. von Elm E, Altman DG, Egger M, Pocock SJ, Gøtzsche PC, Vandenbroucke JP. The Strengthening the Reporting of Observational Studies in Epidemiology (STROBE) Statement: guidelines for reporting observational studies. Int J Surgery (London, England). 2014;12(12):1495-9.

20. Petersen PE, Baez RJ, World HO. Oral health surveys: basic methods. 5th ed ed. Geneva: World Health Organization; 2013.

21. Alshehhi A, Al Halabi M, Hussein I, Salami A, Hassan A, Kowash M. Enamel defects and caries prevalence in preterm children aged 5-10 years in Dubai. Libyan J Med. 2020;15(1):1705633.

22. Pitiphat $W$, Luangchaichaweng S, Pungchanchaikul P, Angwaravong O, Chansamak N. Factors associated with molar incisor hypomineralization in Thai children. Eur J Oral Sci. 2014;122(4):265-70.

23. Lygidakis NA, Dimou G, Marinou D. Molar-incisor-hypomineralisation $(\mathrm{MIH})$. A retrospective clinical study in Greek children. II. Possible medical aetiological factors. Eur Arch Paediatr Dent. 2008;9(4):207-17.

24. Aine $L$, Backstrom MC, Maki R, et al. Enamel defects in primary and permanent teeth of children born prematurely. J Oral Pathol Med. 2000;29(8):403-9.

25. Zhou Y, Lin HC, Lo EC, Wong MC. Risk indicators for early childhood caries in 2-year-old children in southern China. Aust Dent J. 2011;56(1):33-9.

26. Kay EJ, Northstone K, Ness A, Duncan K, Crean SJ. Is there a relationship between birthweight and subsequent growth on the development of dental caries at 5 years of age? A cohort study. Community Dent Oral Epidemiol. 2010;38(5):408-14.

27. Meyer-Lueckel H, Wilson N. Editorial: prevention and treatment of caries simply two sides of a coin. Oral Health Prev Dent. 2018;16(4):305-6.

28. Nirunsittirat A, Pitiphat W, McKinney CM, et al. Adverse birth outcomes and childhood caries: a cohort study. Community Dent Oral Epidemiol. 2016:44(3):239-47.

29. Seow WK. A study of the development of the permanent dentition in very low birthweight children. Pediatr Dent. 1996;18(5):379-84.

30. Ramos SR, Gugisch RC, Fraiz FC. The influence of gestational age and birth weight of the newborn on tooth eruption. J Appl Oral Sci. 2006;14(4):228-32.

31. Gravina DB, Cruvinel VR, Azevedo TD, de Toledo OA, Bezerra AC. Prevalence of dental caries in children born prematurely or at full term. Brazilian oral research. 2006;20(4):353-7.

32. Bastos JL, Peres MA, Peres KG, Barros AJ. Infant growth, development and tooth emergence patterns: a longitudinal study from birth to 6 years of age. Arch Oral Biol. 2007;52(6):598-606.

33. Sharma D, Farahbakhsh N, Shastri S, Sharma P. Intrauterine growth restriction - part 2. J Matern Fetal Neonatal Med. 2016:29(24):4037-48.

\section{Publisher's Note}

Springer Nature remains neutral with regard to jurisdictional claims in published maps and institutional affiliations. 